\title{
Study of Chromosome Abnormalities in Pupils attending Special Schools in Tumakuru District and to correlate with Mental Retardation
}

\author{
Arjun Veeraganahalli Anandappa ${ }^{1}$, Anupama D. ${ }^{1}$, R Lakshmi Prabha Subhash ${ }^{2}$, \\ Satyanarayana $\mathrm{MT}^{3}$, Harshal KL ${ }^{4}$, Jayarama Kadandale ${ }^{5}$, Meenakshi Bhat ${ }^{6}$
}

\author{
${ }^{1}$ Postgraduate, Department of General Medicine, SSIMS \& RC, Davanagere \\ ${ }^{2}$ Professor, ${ }^{3}$ Professor \& HOD, Department of Anatomy \\ ${ }^{3}$ Professor \& HOD, Department of Psychiatry \\ ${ }^{4}$ Research Associate, Cytogenetic Division, ${ }^{5}$ Consultant Cytogeniticist, \\ ${ }^{6}$ Consultant Clinical Geniticist \& Genetic Counselor \\ Sri Siddhartha Medical College \& Research Centre, Tumakuru
}

DOI -10.46319/RJMAHS.2018.v01i01.002

\begin{abstract}
Background: Mental Retardation is defined as a condition of incomplete development of the mind, characterized by impaired developmental skills. Chromosomal abnormalities are known to be an important cause of severe mental retardation. Aim: This study is done to identify the chromosomal abnormalities in pupils of special schools in Tumakuru District \& to correlate it with mental retardation. Materials and methods: With the detailed history \& written consent from the parents, a total number of 25 pupils from special schools in Tumakuru District were subjected to through clinical examination, following which blood samples were obtained and karyotyping was done at cytogenetic laboratory at Department of Anatomy, Sri Siddhartha Medical College, Tumakuru. Results are tabulated following which Genetic counseling was offered to the families of these children. Results: A review was carried out to establish the value of chromosome testing in children with significant developmental delay, where the aetiology was not evident clinically. Of the 25 pupils studied, 7 were girls and 18 were boys. A total of 4 i.e. $16 \%$ of the cases revealed an abnormal karyotype ( 2 boys \& 2 girls). The advantages of chromosome testing in children with developmental delay in whom the etiology is not evident clinically are discussed. Conclusion: The control of genetic diseases should be based on integrated and comprehensive strategy combining best possible treatment and prevention through community education, population screening, genetic counselling \& availability of early diagnosis. Recognition of parents with chromosomal abnormalities which can be inherited, is important, as the risk of recurrence is high in some cases.
\end{abstract}

Key words: Chromosome abnormalities, mental retardation (MR), special schools, genetic counseling, developmental delay (DD)

\section{Introduction}

Mental Retardation is defined as a condition of arrested or incomplete development of the mind, characterized by impaired developmental skills that contribute to the overall level of intelligence. ${ }^{[1]}$ Chromosomal abnormalities are known to be an important cause of severe mental retardation which affects about $1-3 \%$ of the population. The highest incidence is in school age children, with the peak at ages 10-14 yrs. It is more common among men than in women. Chromosomal abnormalities that alter developmental gene expression are the most important cause of mental retardation.$^{[1]}$
Children with developmental delay will usually be seen at an early age for pediatric assessment if associated with evident dysmorphic features and, it may also include cytogenetic analysis. ${ }^{[2]}$ Biological abnormalities can be detected in approximately $25 \%$ of the cases of mental retardation on extensive investigations, which can be recognizable at birth or in early childhood. ${ }^{[3]}$ Mild developmental delay occurring in children without

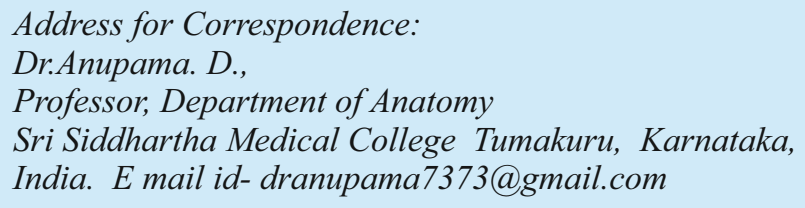


prominent clinical features, however, is not usually considered, for chromosomal analysis. Such children may do reasonably well in preschool age and are often referred to special schools after a period in normal schools, when their difficulty in coping becomes apparent. A cytogenetic survey in them will give us the clue regarding the etiology of the condition. Special schools provide education for children who come in the range of mild mental retardation, defined by the World Health Organization as IQ 50-70. Diagnosis of Mental retardation can be made after the history, a standard IQ test and a measure of adaptive functions. IQ tests are usually carried out only in children who have been failed academically and are being considered for special schools. Factors other than IQ like social adaptation, parental attitudes may also contribute and the availability of remedial teaching in the 'normal' school can all bias selection. Thus a study population of children attending special schools is unlikely to contain all school children in the IQ range 50-70, nor is it unbiased. With these limitations, this study is considered a valid project and only way to identify mildly mentally retarded children, as the contribution of chromosomal abnormalities in etiology of mental retardation is high. ${ }^{[4]}$ In 1800 s, the notion that mental retardation was based primarily on a deficit in social or moral reasoning. ${ }^{[1]}$ Down syndrome is also known as trisomy 21, trisomy $G$ and mongolism. The first description of a child who presumably had Down syndrome was provided by Esquirol in $1838 .{ }^{[5]}$ In 1866 John L. Down published a paper accurately describing some of the characteristics of this syndrome which today bears his name. In 1959, Lejeune and Jacobs independently determined that Down syndrome was caused by Trisomy $21 .{ }^{[6]}$ Down syndrome is an easily recognized congenital, autosomal (non-sex chromosomes) anomaly commonly characterized by generalized growth deficiency and mental deficiency affecting 1 in 600 to 1 in 1000 live births. ${ }^{[7]}$ Approximately $95 \%$ of Down syndrome cases have extra chromosome 21, making the chromosome count 47 instead of the normal 46 . The other $5 \%$ are accounted for by other chromosomal abnormalities including translocation $(3 \%)$ and mosaicism $(2 \%)$ or partial trisomy. Chromosomal abnormalities are responsible for up to $28 \%$ of all mental retardation cases (Curry et al., 1997) ${ }^{[8]}$ Fragile $\mathrm{X}$ is the second most common cause of mental retardation. ${ }^{[9]}$ A cat-like cry in infants with Cri-du-Chat (Cornish, Munir, \& Bramble, 1998), Patau syndrome and some syndromes associated with distinctive features such as obsessive eating in Prader Willi are also associated with mental retardation (Dykens \& Cassidy, 1999; Russell \& Oliver, 2003). ${ }^{[10,11]}$

\section{Aims and objectives}

This study was done to identify the chromosomal abnormalities in pupils of special schools in Tumakuru district \& to correlate it with mental retardation. This also surveys the children with mild mental retardation who have shown a higher prevalence of chromosome abnormalities than that exists in the general population. Chromosomal analysis in this survey was not limited to the mildly retarded children with dysmorphic features, but also includes children with only slow mentation thus investigating the etiology of mild mental handicap. This study may also aid in early intervention and a full needs assessment of the children which is necessary for treatment, care \& support.

\section{Materials and methods:}

Chromosome analysis is performed as an investigation to know the etiology of the condition.

With the detailed history \& written consent from the parents, a total number of 25 pupils from special schools in Tumakuru District were subjected to through clinical examination, following which blood samples were obtained and karyotyping was done. Information collected for each child also included details of pre- and peri natal history and early development and they were categorized as mild, moderate and severe mental retardation cases. A subjective assessment was made about the social conditions at home and parental ability; these were classed as high, middle \& lower. Blood samples obtained were subjected for karyotyping at cytogenetic laboratory of Department of Anatomy, Sri Siddhartha Medical College, Tumakuru. Cultures were set up in RPMI medium and harvested for 72 hours. Metaphases with $\mathrm{G}$ banding staining techniques will be examined from each child. Parents' chromosomes will be analyzed when a possible familial abnormality is found. Results from a total population of 25 children from different special schools were tabulated.

\section{Results:}


A review was carried out to establish the value of chromosome testing in children with significant developmental delay, where the aetiology was not evident clinically. Of the 25 pupils studied, 7 were girls and 18 were boys. A total of 21 i.e. $84 \%$ of the cases revealed a normal karyotype ( 16 boys $\& 5$ girls), 4 i.e. $16 \%$ of the cases revealed an abnormal karyotype ( 2 boys $\& 2$ girls) as shown in Figure 1. 16 children had dysmorphic features; 3 (25\%) of these had an abnormal karyotype. Normal male karyotype is shown in Figure 2. There were 3 Down's syndrome cases with Trisomy 21 in 2 ( 1 boy \& 1 girl) as shown in figure $3 \&$ Trisomy 21 with translocation, 47, XY, der(15;21), (q10;q10) +21 in 1(boy) as shown in Figure 4. Another girl had a balanced translocation with karyotype $45, \mathrm{XX}$, der $(14 ; 21)(\mathrm{q} 10 ; \mathrm{q} 10)$ as shown in Figure 5 , who was one of the 25 who had no dysmorphic features, but had a chromosome abnormality. In this case, the etiology for severe mental retardation had to be sought. All twenty-

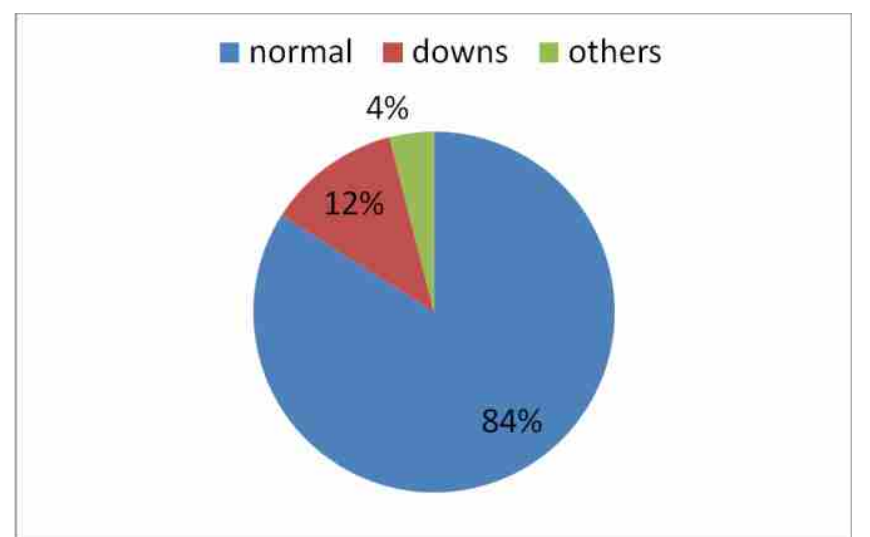

Figure 1. Population percentage with normal \& abnormal Karyotypes

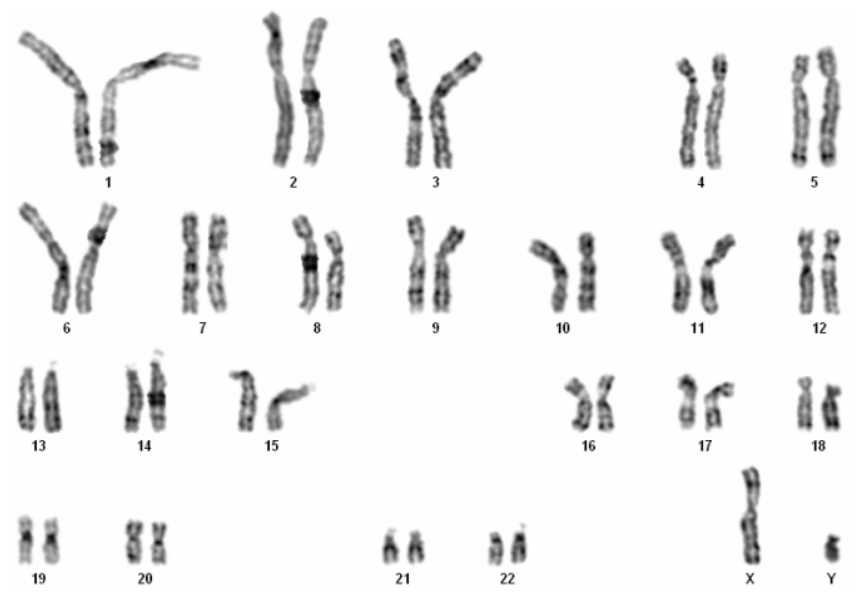

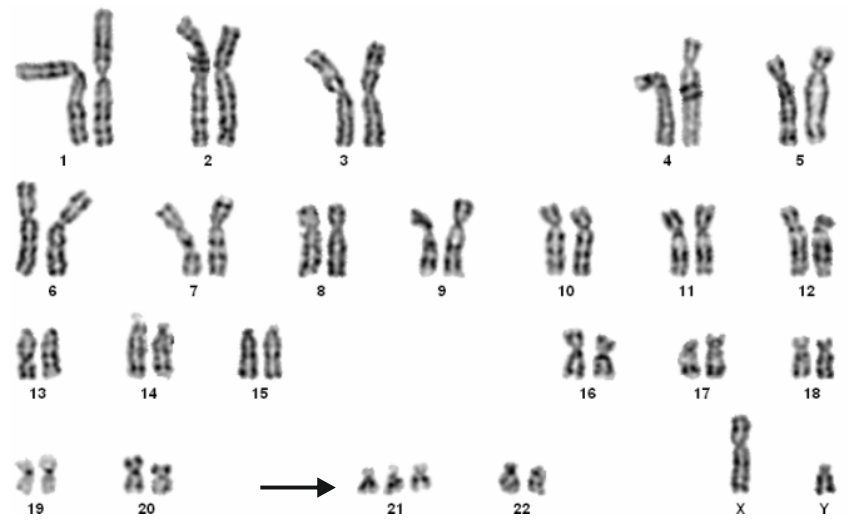

Figure 3. Free Trisomy 21

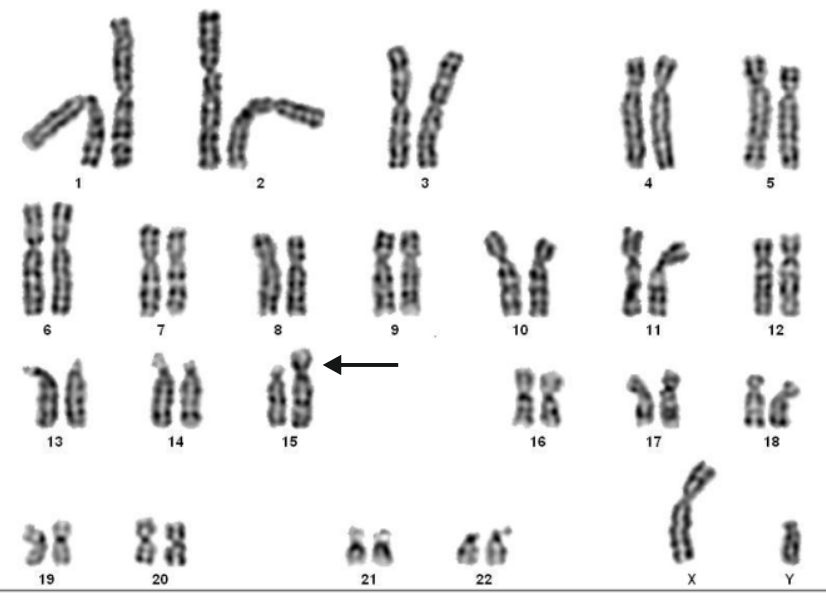

Figure 4 : Trisomy 21 with translocation $[47, \mathrm{XY}, \operatorname{der}(15 ; 21),+21]$

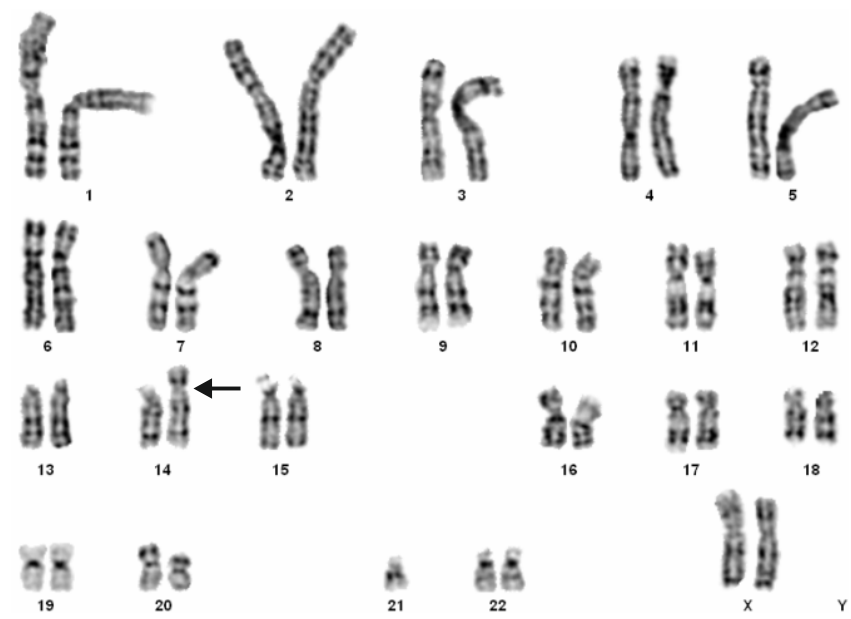

Fig 5: Balanced Tranlocation 45, XX, $\operatorname{der}(14 ; 21)(\mathrm{q} 10 ; \mathrm{q} 10)$

five children had intellectual disability; 4 (16\%) of these had an abnormal karyotype. The advantages of chromosome testing in children with developmental delay in whom the aetiology is not evident clinically are

Figure 2. Normal Male Karyotype 46,XY 
discussed.

\section{Discussion:}

There is a standard recommendation that chromosomes be obtained in any patient who presents with developmental delay (DD) or mental retardation (MR) regardless of whether or not they have dysmorphic features. ${ }^{[6]}$ Increasingly, if patients are physically wellformed, the option to perform a karyotype is questioned because of the presumed low yield of a chromosomal abnormality. We hypothesize that patients with DD/MR who are non-dysmorphic do not have abnormal chromosomes at a rate high enough to warrant obtaining a karyotype on all patients in this population. ${ }^{[7,10]}$ The total number of subjects selected for the study was 25 . All these patients were recommended to have chromosome testing. Of these, 21 had normal karyotype. Three subjects were found to have Down syndrome, accounting for $12 \%$ of the cases. One subject had a balanced translocation 21 to 14 . Our yield of chromosome analysis in non-dysmorphic patients with $\mathrm{DD} / \mathrm{MR}$ is less than that previously described. Several surveys have reported the frequency and types of chromosomal abnormalities in patients with mental retardation. The most common chromosomal abnormality among patients in institutions is Down's syndrome which coincides with our study results. It is said to be the most commonest genetic cause of Mental retardation accounting for one third to one fourth of the cases. ${ }^{[8,}{ }^{9]}$ In cases with Down syndrome and translocations, the parents should be councelled, for recurrence risk, advised prenatal diagnosis for future pregnancies if family is not competed. The incidence of chromosome abnormalities has increased by 10 fold, which can be detected with less sophisticated cytogenetic techniques, even in newborns. If the chromosome analysis is done only to children with dysmorphic features, we will fail to identify four to five of the six children which can be identified during this study although there is no clear indication for chromosomal analysis other than mild mental retardation. The study may also detect chromosome abnormality which is inherited from phenotypically normal parents. It is possible to identify problems in the early history and family, responsible for the early referral to a special school during the survey.

\section{Conclusion:}

The control of genetic diseases/chromosomal abnormalities should be based on integrated and comprehensive strategy combining best possible treatment and prevention through community education, population screening, genetic counselling \& availability of early diagnosis. Among a group of pupils in special schools where the karyotyping was done, the autosomal aberrations were seen. Trisomy 21 was the most frequent of all. Recognition of parents with chromosomal abnormalities is important, as the risk of recurrence is high in some cases. This knowledge allows for proper genetic counselling. Although chromosomal abnormalities cannot be corrected, screening parents for carrier status of certain genetic abnormalities can result in reduced incidence of the condition. Genetic counseling of patients with various chromosomal defects \& their parents, helps in guiding them for proper clinical management. A history of consanguinity, between close relatives increases the chance of a child having a genetic disorder because both parents are more likely to have the same abnormal genes, the risk of which can also be brought down by premarital investigations of the partners and also prenatal diagnosis in future pregnancies.

Financial support and sponsorship: ICMR short term student (STS) project

\section{Conflicts of interest: Nil}

\section{Acknowledgement}

The author wish to thank ICMR and Dr A G Srinivasa Murthy, Principal, SSMC for his constant support and guidance, the pupils and their parents and Heads of special schools

\section{References:}

1. Mental retardation: In Kaplan Sadock's Synopsis of Psychiatry.10th ed (South Asian ed). Lippincott Williams Wilkins Publications. Ch. 38. p 1138-57.

2. M A Lamont, $N$ R Dennis, and M Seabright. Chromosome abnormalities in pupils attending ESN/M schools. Archives of Disease in Childhood, 1986, 61, 223-226.

3. Mental retardation R.K.Chadda: In Text book of Post graduate Psychiatry. Vyas \& Ahuja. 2008. vol 2,Ch.38, p572- 85.

4. Puteri Jamilatul Noor. Y M Chin. S K Ten, Khalid Hassan. Prevalence of chromosomal anomalies of the mentally retarded-Report of a study of 124 institutionalized children in Kaulalumpur. vol.28, No.3, 
JUNE 1987.

5. Pueschel SM. A historical view point. In: Pueschel SM, ed. Down syndrome growing and learning. (Human potentials for children series). : Canning, Murphy, Zaumer, Andrews and McMeel, Inc. p. 37-9,1981.

6. Smith W.B. Recognizable Pattern of Human Malformations. Fourth ed. Jones LK, ed. Philadelphia: W.B. Saunders Co; 1988. p. 10-12.

7. Korenberg JR., Pulst SM., Gerwehrs. Advances in the understanding of chromosome 21 and Down Syndrome. In: Lott I, McCoy E, editors. Down syndrome: Advances in medical care. New York: Wiley-Liss; 1992. p. 3-12.

8. Usha Dave and Dhanalaxmi shetty. Chromosomal abnormalities in Mental retardation: Indian experience. Int J Hum Genet, 10(1-3):21- 32 (2010)

9. Modesto AM, Aguiar Mde F, Barbosa H, Vilela SS,
Santos M, Ferrari I, Klotz M Fragile X Syndrome: case report in two brothers. J Pediatr (Rio J). 1997 NovDec;73(6):419-22.

10. D.Armalina, TI. Winarni, Farmaditya EP Mundhofir, A.Purwanti, Sultana MH Faradz Phenotype and cytogenetic profile of Down syndrome cases in special schools in semarang In: International Seminar and Workshop on Modern Biology and its applications: Focusing on Stem Cells and Human Genetics, 28-30 November 2009, Semarang, Indonesia.

11. R.Jayasekara .Chromosome anomalies in Srilanka:A cytogenetic profile .Ceylon J Med.Sci., 31 (No.1,June) 1998, pp 1-5 